\title{
A randomised controlled study comparing bromocriptine to which levodopa was later added, with levodopa alone in previously untreated patients with Parkinson's disease: a five year follow up
}

\author{
J L Montastruc, O Rascol, J M Senard, A Rascol
}

\begin{abstract}
This pilot study was performed to compare the occurrence of long term motor complications in Parkinson's disease when the introduction of levodopa was delayed by an initial treatment with high doses of bromocriptine alone. The trial was a prospective randomised controlled study comparing 31 previously untreated patients with Parkinson's disease initially given bromocriptine alone to which levodopa was later added (group B/D) and 29 other previously untreated patients with Parkinson's disease immediately given levodopa alone (group D). The end point was the occurrence of the first motor complications (wearing off or dyskinesia). Group B/D patients received bromocriptine (52 (SEM 5) $\mathrm{mg} / \mathrm{day}$ ) for 2.7 years, to which levodopa was later added (471 (SEM 46) mg/day). Group D patients received a comparable dose of levodopa alone (569 (SEM 47) mg/day). Both had similar disability scores at the end of the study. Motor complications were fewer and appeared later in group B/D than in group D (56\% after 4.9 (SEM $0 \cdot 5)$ years of treatment $v 90 \%$ after 2.7 (SEM 0.5) years, $p<0.01$ ). Wearing off appeared later $(p<0.01)$ in group $B / D$ (4.5 (SEM 0.6) years) than in group $D$ (2.9 (SEM 0.6) years). Peak dose dyskinesia occurred less often in group $B / D$ patients (three $v 14$ cases, $p<0.01$ ). This study showed that a three year initial monotherapy with high doses of bromocriptine followed by addition of levodopa delayed the occurrence of long term motor complications usually found in patients with Parkinson's disease treated with levodopa alone from the beginning.
\end{abstract}

\section{(F Neurol Neurosurg Psychiatry 1994;57:1034-1038)}

Levodopa remains the gold standard in the treatment of Parkinson's disease. Long term levodopa treatment, however, leads to the occurrence of late side effects such as abnormal involuntary movements or motor fluctuations. ${ }^{12}$ Several putative risk factors have been proposed to explain these side effects- namely, the age of onset of Parkinson's disease, ${ }^{34}$ the severity and duration of the disease, ${ }^{5-7}$ and the duration and dose of levodopa. ${ }^{8}$ Most of these factors are closely related and none can be considered independently of the others when treating the patients. Consequently, most of the retrospective studies available have produced contradictory conclusions and there is still a large controversy about the advantages and disadvantages of early or late treatment with levodopa. ${ }^{9-11}$

Early combination of a dopamine agonist with levodopa has been claimed to reduce the occurrence of fluctuations and peak dose dyskinesia $^{1213}$ although this conclusion was recently disputed. ${ }^{1415}$ We previously reported that a three year treatment of previously untreated patients with Parkinson's disease with a dopamine agonist alone (bromocriptine) does not induce the motor side effects often found with levodopa. ${ }^{16} 17$ The long term use of bromocriptine monotherapy, however, is limited by a decline in efficacy or the occurrence of non-motor side effects (hallucinations, oedema, fibrosis)..$^{21617}$ In these situations, it becomes necessary to add levodopa to bromocriptine or to completely replace bromocriptine by levodopa. There are no controlled data available about the long term follow up of patients treated according to such a strategy. In other words, prospective data are lacking to assess whether delaying levodopa by an initial treatment with a dopamine agonist alone delays the occurrence of motor complications or not.

The objective of the present prospective randomised controlled pilot trial was to compare the occurrence of long term motor complications in two groups of previously untreated patients with Parkinson's disease: a group treated with bromocriptine alone for as long as possible to which levodopa was later added (group B/D) $v$ a group receiving levodopa alone (group D), with a five year follow up.

\section{Patients and methods}

The study included 60 patients who met the United Kingdom Parkinson's Disease Society Brain Bank clinical diagnostic criteria for idiopathic Parkinson's disease. ${ }^{18}$ Patients with 
Table 1 Main characteristics of the patients at entry into the study

\begin{tabular}{lll}
\hline & Group B/D & Group D \\
\hline Patients & 31 & 29 \\
Men & 19 & 17 \\
Women & 12 & 12 \\
Mean age (y) & $61(2)$ & $62(2)$ \\
Disease duration (y) & $2 \cdot 7(0 \cdot 3)$ & $2 \cdot 7(0 \cdot 4)$ \\
Hoehn and Yahr stage: & 12 & 12 \\
I & 18 & 13 \\
II & 1 & 4 \\
III & 1 & \\
\hline
\end{tabular}

Values in parentheses are SEM. There were no significant differences between the groups.

clinical features suggestive of progressive supranuclear palsy ${ }^{19}$ or multiple system atrophy $^{20}$ were excluded as well as patients with "juvenile" parkinsonism ( $<20$ years of age) as it has been suggested that they have a separate disease. Patients with young onset Parkinson's disease were accepted ( $>30$ years).$^{21}$ Patients older than 75 years at the onset of Parkinson's disease were also excluded because we wanted a long term follow up. No patients had previously received antiparkinsonian drugs such as levodopa, dopamine agonists, or selegiline. Amantadine and anticholinergics, if prescribed, were stopped when the patients entered the trial.

The patients were randomly assigned to two different pharmacological strategies: (a) initial treatment with bromocriptine alone to which levodopa was later added (group B/D) or (b) levodopa alone from the start (group D). Antiparkinsonian drugs other than bromocriptine and levodopa were not used during the study. Bromocriptine and levodopa (plus dopa decarboxylase inhibitor) were introduced at low dosages $(2.5$ and $50 \mathrm{mg}$ respectively) in the form of three nearly equal doses per day that were gradually increased over a three to six week period according to the patient's response. Levodopa and bromocriptine were used all through the study at the lowest doses able to induce sufficient functional improvement as judged by the patient and the physician. High doses of bromocriptine were used as the maximum allowed dose was $90 \mathrm{mg} /$ day. Domperidone $(60 \mathrm{mg} /$ daily) was used when needed to prevent nausea, vomiting, or orthostatic hypotension. In group $\mathrm{B} / \mathrm{D}$, levodopa was added to bromocriptine when a decline in efficacy of the maximal tolerated dose of bromocriptine occurred or when bromocriptine-induced side effects necessitated lowering the dose, with consequent motor deterioration. Any change in any drug dose was fully documented.

Table 1 presents main demographic data and clinical features of the patients of group $B / D$ and group $D$ (age, sex, duration of the disease, clinical stage on entry into the protocol according to the Hoehn and Yahr scale)..$^{22}$ There were no significant differences between the two groups. Most patients were at clinical stages I or II when they entered the study.

Patients entered the study from 1982 to 1989. Each of the 60 patients was seen regularly twice a year by the same physician (one of the four authors) throughout the study.
The motor status was assessed with the Columbia University scale until 1985 and later with the newly developed Unified Parkinson's disease rating scale (UPDRS). ${ }^{23}$

The end point of the study was defined as the moment when the first motor complication occurred. Two different types of motor complication were defined: the first was involuntary abnormal movements-that is, peak dose or biphasic dyskinesia or dystonia. ${ }^{24}$ The second was motor fluctuation-that is, wearing off or "on-off" phenomena. ${ }^{24} 25$ The occurrence of each motor side effect was detected both by questioning the patient and by observation. When detected, each motor complication was documented by an objective observation by the physician on the next two visits, at a one month interval.

All patients continuing in the study (those who had not yet reached the end points) were reviewed in October 1992. The mean follow up was similar in the two groups (5.4 (SEM 0.5 ) years in group B/D and 4.9 (SEM 0.6 ) years in group D).

Comparisons were performed by one way analysis of variance followed by MannWhitney $U$ test or (as required) Student's $t$ test for paired comparisons. The frequency of motor complications was compared between the two groups with the $\chi^{2}$ test with Yates' correction. The probability of keeping free of motor complications was assessed as a function of time according to the Kaplan-Meier product limit estimation. ${ }^{26}$ The comparison between the two groups was carried out by a log rank test. ${ }^{27}$ An attempt to test the difference through the Cox model was given up when the proportional hazard hypothesis proved irrelevant. A relative risk was calculated from the Mantel-Haenszel estimator modified by Tarone. ${ }^{28}$ The results are mean values (SEM).

\section{Results}

All the patients included were taken into account, except five in the group B/D. In this group, four patients were still on bromocriptine alone and did not require levodopa adjunction on the last follow up visit (October 1992). None of them presented motor complications. The fifth patient had severe hallucinations after six months of bromocriptine alone $(50 \mathrm{mg} / \mathrm{day})$. She was then treated with levodopa alone $(600 \mathrm{mg} /$ day $)$ and developed the same complication within three months. She refused to come back to the following visit. She was the only patient lost in the study.

The number of patients reaching the end point when the study terminated was significantly different between the two groups (table 2): 14 patients in group $B / D$ and 26 in group $D(p<0.01)$. The smaller number of motor complications in the $B / D$ group was due to a significantly smaller number of peak dose dyskinesias (three in group $\mathrm{B} / \mathrm{D} v 14$ in group $\mathrm{D}, \mathrm{p}<0.01$ ). One patient in group $\mathrm{B} / \mathrm{D}$ and two in group $\mathrm{D}$ had peak dose dystonias. The number of occurrences of wearing off was 
Table 2 Comparison of number of patients reaching the end points and of delay to reach the end points

\begin{tabular}{|c|c|c|c|}
\hline & $\begin{array}{l}\text { Group B/D } \\
(n=25)\end{array}$ & $\begin{array}{l}\text { Group D } \\
(n=29)\end{array}$ & p Value \\
\hline \multicolumn{4}{|l|}{ Number of patients reaching: } \\
\hline end point (motor complications) & 14 & 26 & $<0 \cdot 01$ \\
\hline peak dose dyskinesias & 3 & 14 & $<0.01$ \\
\hline peak dose dystonias & 1 & 2 & NS \\
\hline wearing off & 10 & 10 & NS \\
\hline \multicolumn{4}{|l|}{ Delay to reach end-point (mean (SEM)): } \\
\hline from diagnosis & $8 \cdot 1(0 \cdot 7)$ & $5 \cdot 4(0 \cdot 7)$ & $<0.01$ \\
\hline from first treatment & $4.9(0.5)$ & $2 \cdot 7(0.5)$ & $<0.01$ \\
\hline from levodopa & $2 \cdot 2(0.4)$ & $2 \cdot 7(0.5)$ & NS \\
\hline \multicolumn{4}{|l|}{ Delay to reach wearing off (mean (SEM)): } \\
\hline from diagnosis & $7 \cdot 9(1 \cdot 0)$ & $6 \cdot 2(0.9)$ & NS \\
\hline from first treatment ${ }^{*}$ & $4 \cdot 5(0 \cdot 6)$ & $2 \cdot 9(0 \cdot 6)$ & $<0.01$ \\
\hline from levodopa & $2 \cdot 1(0 \cdot 5)$ & $2.9(0.6)$ & NS \\
\hline
\end{tabular}

"first treatment = bromocriptine in group B/D and levodopa in group " $\mathrm{D}$ ".

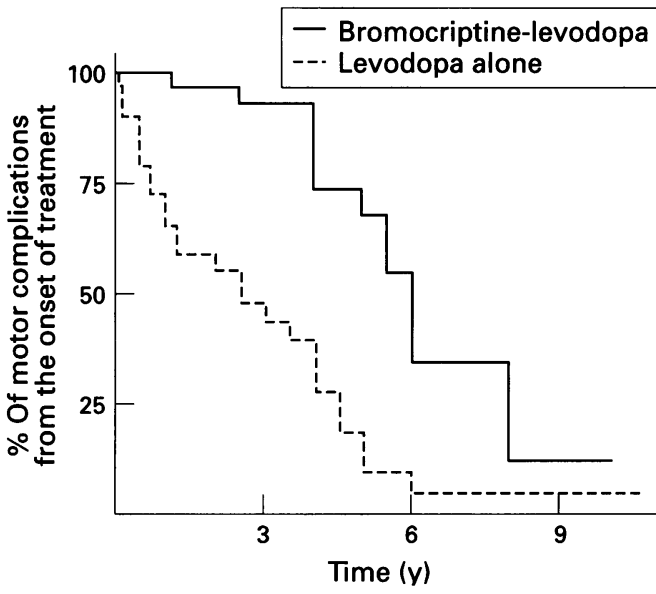

Figure 1 Cumulative probability of reaching the end point (Kaplan-Meier estimate): percentage of patients free of motor complications from the onset of treatment according to treatment group. For details see text.
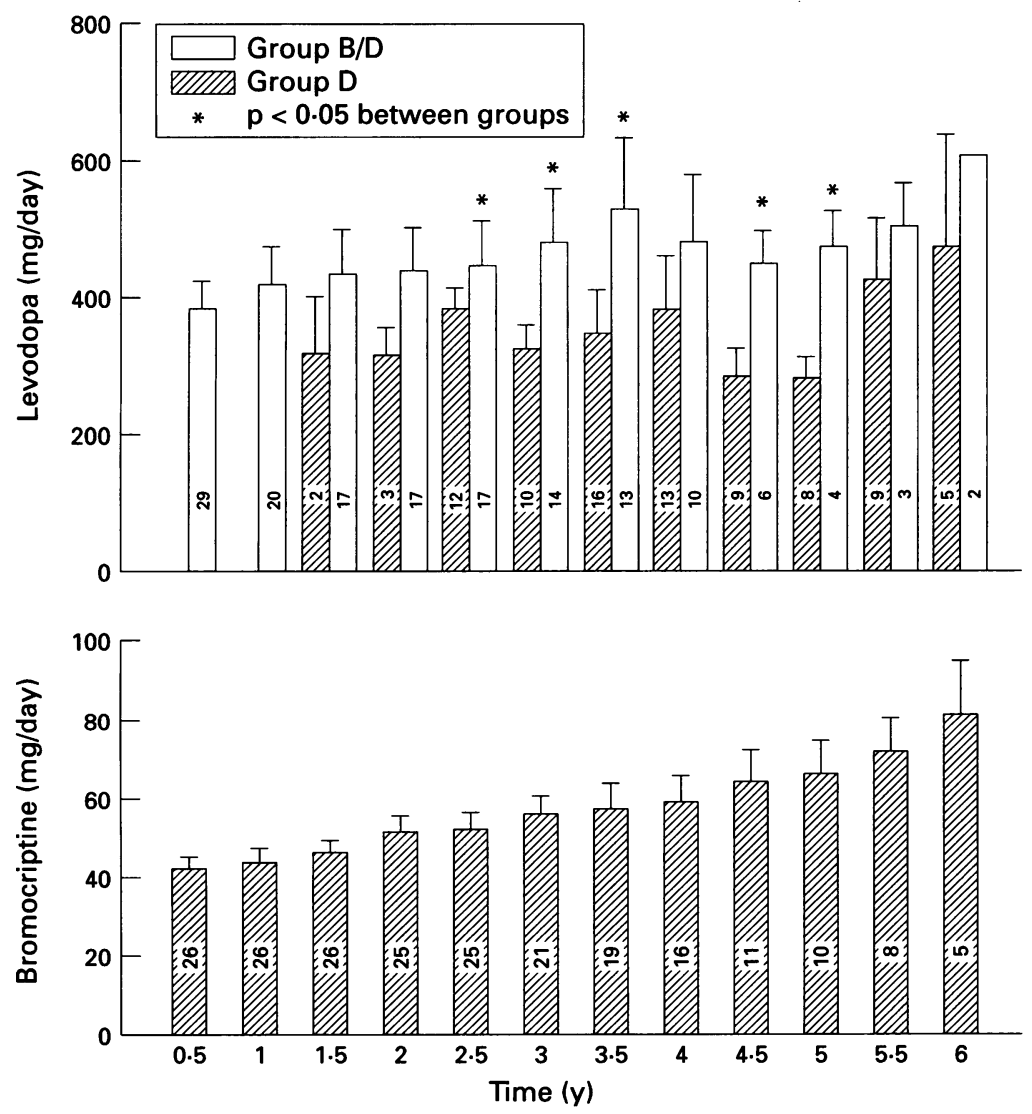

Figure 2 Mean (SEM) doses of bromocriptine and levodopa in the two groups of patients during the first six years of the study. The number of patients continuing the study is indicated in the bars for each period. similar in both groups $(n=10)$. We did not find unpredictable on-off phenomena, biphasic dyskinesias, or freezing when walking as first motor complications in this study.

Table 2 also shows that the time taken to reach the end points was longer in group $B / D$ than in group $\mathrm{D}$ whether it was measured from the time of diagnosis or from the initiation of the first treatment (bromocriptine in group $\mathrm{B} / \mathrm{D}$ or levodopa in group $\mathrm{D}$ ). Conversely, the delay was similar in both groups when measured from levodopa introduction. The differences were mainly due to differences in the time taken for wearing off to begin.

Kaplan-Meier plots (fig 1) of the probability of not reaching the end point in group $B / D$ and $\mathrm{D}$ differed widely as proved by the $\log$ rank test $\left(\chi^{2} 15.9, \mathrm{df}=1 ; \mathrm{p}<0.001\right)$. The Mantel-Haenszel estimation modified by Tarone indicated a relative risk of $1 / 4 \cdot 21=$ 0.24 in developing motor complications when bromocriptine was prescribed before levodopa.

Motor complications were not the only side effects during the study. Seven patients had psychiatric side effects (hallucinations or confusion) before the occurrence of motor end points: five in group B/D with $50 \mathrm{mg}, 60 \mathrm{mg}$, $30 \mathrm{mg}$ (plus $600 \mathrm{mg}$ levodopa), $60 \mathrm{mg}$ (plus $250 \mathrm{mg}$ levodopa), and $40 \mathrm{mg}$ (plus $400 \mathrm{mg}$ levodopa) bromocriptine. Bromocriptine was withdrawn in three cases. The dose was decreased by $50 \%$ in the two other patients. In these five cases, the hallucinations disappeared and the follow up was continued until a motor end point was reached. Two patients of group $\mathrm{D}$ also had hallucinations with levodopa monotherapy before developing motor complications (350 and $400 \mathrm{mg} /$ day) Levodopa was continued as it was not possible to reduce the dose because of the motor impairment. No neuroleptic treatment was prescribed. Clozapine was not available for this indication in France at this time.

Figure 2 shows the mean doses of levodopa and bromocriptine at each six monthly visit. The daily dose of levodopa was significantly higher in group $\mathrm{D}$ than in group $\mathrm{B} / \mathrm{D}$ from the 30 th month of treatment. The mean doses of levodopa and bromocriptine in patients with or without motor complications were not different (data not shown).

There was no difference between the two groups in the severity of the disease assessed with the Hoehn and Yahr scale when the patients entered the study. At the end point or the last visit of follow up, the UPDRS motor scale showed no significant difference between the two groups $(10.6(1 \cdot 1)$ in group $\mathrm{B} / \mathrm{D} v 11.0(1.5)$ in group " $\mathrm{D}$ ").

\section{Discussion}

The study shows that motor complications (wearing off and peak dose dyskinesia) occurred later and were less numerous in the group treated with high doses of bromocriptine followed by levodopa than in patients treated with levodopa alone from the start. In 
patients treated with levodopa, motor side effects were detected in $90 \%$ of the cases after three years of levodopa monotherapy. This is rapid compared with other studies. ${ }^{29-31}$ The difference is probably explained by the fact that we carefully and prospectively followed up our patients and thus often detected mild motor complications before they were noticed by the patients themselves. In contrast, motor complications occurred in $56 \%$ of the patients in group $\mathrm{B} / \mathrm{D}$. Nakanishi et al ${ }^{32}$ described the occurrence of late motor side effects in $49 \%$ of patients treated with levodopa and $36 \%$ of $\mathrm{B} / \mathrm{D}$ group. In their study, however, the dosage of bromocriptine was lower (mean value around $12.5 \mathrm{mg}$ ) than in the present work and no statistical evaluation was presented in their report.

The present trial also found that delaying levodopa with bromocriptine did not influence wearing off and peak dose dyskinesia in a similar way. In fact, the number of patients with wearing off was similar in groups $B / D$ and $D$, but it occurred later when levodopa was given late (group $B / D$ ). Conversely, the frequency of dyskinesia was significantly lower in group $\mathrm{B} / \mathrm{D}$ - that is, when levodopa was delayed. Our result is not in accordance with the data of Nakanishi $e t a^{32}$ who described a similar frequency of dyskinesia in both groups $(8.5 \%$ in $\mathrm{B} / \mathrm{D}$ and $10 \%$ in $\mathrm{D})$ after five years of treatment. They also found the same frequency of occurrence for on-off and wearing off phenomena in both groups.

The results of the present study suggest that wearing off phenomena and peak dose dyskinesias have different pathophysiological mechanisms. ${ }^{29-31}$

Dyskinesia occurred significantly less often in the $\mathrm{B} / \mathrm{D}$ group than in the $\mathrm{D}$ group. It has been shown that bromocriptine alone does not induce dyskinesia in previously untreated patients with Parkinson's disease ${ }^{161733}$ or in the primate MPTP model of parkinsonism. ${ }^{34}$ The incidence of dyskinesia is also reduced when bromocriptine or levodopa are given together early in the course of treatment. ${ }^{12} 13$ This conclusion was recently discussed by Weiner's group. ${ }^{1415}$ The few patients in their study (10 in the levodopa group and seven in the early combination group) does not allow any definite conclusions to be reached. Our results show that the occurrence of dyskinesias was lower when the drugs were given together later. Several hypotheses can be proposed to explain this. It is possible that the more sustained dopaminergic stimulation elicited by bromocriptine reduces the occurrence of dyskinesia. It is also possible that the difference can be explained by the D1 antagonist effect of bromocriptine or its inability to discriminate between the high and low affinity states of the D2 receptor. In our study, however, the symptomatic effect of bromocriptine allowed us to use lower doses of levodopa in the $B / D$ group than in the $D$ group. This difference may also explain our results as reducing the levodopa dose is the best way to reduce the intensity of dyskinesia.

Long term motor complications appeared after the same duration of treatment with levodopa in both groups. This suggests that the late motor complications encountered in patients with Parkinson's disease are indeed more closely related to the duration of the treatment with levodopa than to the evolution of the disease. Whether this difference can also be explained by the putative protective effect of the dopamine agonist as suggested by "in vitro studies" 35 remains a topic for future research. A recent multicentre study showing that bromocriptine reduced the risk of mortality in patients treated with levodopa (by more than $50 \%$ ) has suggested a cardioprotective effect of bromocriptine. ${ }^{36}$ In our study, one patient in the $B / D$ group and three in the $D$ group died in October 1992. Our population is too small and our follow up too short to allow any conclusions on this point.

We make two other comments about the present trial. Firstly, we prospectively followed two cohorts of previously untreated patients with Parkinson's disease who were randomly assigned to two different therapeutic regimens as soon as a dopaminergic treatment was considered necessary. By this means, most of the risk factors putatively influencing the occurrence of long term motor side effects were not significantly different between the two groups. The age at onset of disease was similar. The severity and the duration of the disease were similar when starting the treatment. The symptomatic improvement induced by both therapeutic strategies was similar at the end of the study. Therefore, we can assume that the differences in patient outcomes is mainly related to the difference in drug regimen. It is true, however, that the present study has a methodological limit because it was not blind. In fact, this trial must be considered as a long term pilot study as it was designed in the early 1980 s when such a strategy had not been tested before. Since that time, blind studies built on similar patterns have been started with other dopamine agonists but have several years to run before their results become available.

Secondly, it is likely that a proportion of the patients enrolled in our study had extrapyramidal syndromes other than idiopathic Parkinson's disease. In fact, when reviewing the 60 cases in October 1992, two patients of the group $\mathrm{B} / \mathrm{D}$ and two patients of the group D subsequently presented atypical features such as dementia, or pyramidal, dysautonomic, or cerebellar symptoms that make the diagnosis of idiopathic Parkinson's disease questionable. All of them, however, initially responded to dopaminergic drugs. The fact that we randomly allocated the treatments suggests that the same proportion of atypical Parkinson's disease should have been included in each arm of the study so that our results are still valid despite the methodological limits.

In conclusion, this study shows that a three year initial monotherapy with high doses of bromocriptine followed by a late use of levodopa as an adjunct delays the occurrence of long term motor complications usually found 
in patients with Parkinson's disease treated with levodopa alone from the beginning. Further studies must be performed to compare the results of this new strategy (late levodopa combination treatment with a dopamine agonist) with other therapeutic schemes.

We thank Doctor JP Charlet (Department of Medical Computation and Statistics, University Hospital, Toulouse, France) for his help in the statistical analysis, the nurses of the Unit of Clinical Neuropharmacology (Hopital Purpan, Toulouse), and Mrs Bontemps for the preparation of the manuscript.

1 Marsden CD, Parkes JD. "On-off" effects in patients with Parkinson's disease on chronic levodopa therapy. Lancet 1976;i:292-6.

2 Montastruc JL. Recent advances in the clinical pharmacology of Parkinson's disease. Therapie 1991;46:293-303.

3 Cedarbaum JM, Gandy SE, McDowell FH. "Early" initia tion of levodopa treatment does not promote the development of motor response fluctuations, dyskinesias, or dementia in Parkinson's disease. Neurology 1991;41: 622-9.

4 Peppe A, Dambrosia JM, Chase TN. Risk factors for motor response complications in L-dopa-treated parkinsonian patients. In: Narabashi $\mathrm{T}$, Nagatsu $\mathbf{N}$ Yanagisawa N, Mizuno, Y, eds. Advances in Neurology. New York: Raven Press Ltd, 1993, 698-702.

5 Horstink MWIM, Zijlmans JCM, Pasman JW, et al. Which risk factors predict the levodopa response in fluctuating risk factors predict the levodopa response in fluctur

6 Caraceni T, Scigliano G, Musicco M, et al. The occurrence of motor fluctuations in parkinsonian patients treated long-term with levodopa: role of early treatment and disease term with levodopa: role of early treat.

7 Horstink MWI, Zijlmans JCM, Pasman JW, et al. Severity of Parkinson's disease is a risk factor for peak-dose dyskinesia. F Neurol Neurosurg Psychiatry 1990;53:224-6.

8 De Jong GJ, Meerwaldt JD, Smitz PIM. Factors that influence the occurrence of response variations in Parkinson's disease. Ann Neurol 1987;22:4-7.

9 Fahn S, Bressman B. Should levodopa therapy for parkinsonism be started early or late? Evidence against early treatment. Can $₹$ Neurol Sci 1984;11:200-6.

10 Melamed E. Initiation of levodopa therapy in parkinsonian patients should be delayed until the advanced stages of patients should be delayed until the ad

11 Markham C, Diamond S. Modification of Parkinson's disease by long-term levodopa treatment. Arch Neurol 1986;43:405-7.

12 Rinne UK. Combined bromocriptine-levodopa therapy early in Parkinson's disease. Neurology 1985;35:1 196-8.

13 Rinne UK. Early combination of bromocriptine and levodopa in the treatment of Parkinson's disease: a 5-yea follow-up. Neurology 1987;37:826-8.

14 Weiner WJ, Factor SA, Sanchez-Ramos JR, et al. Early combination therapy (bromocriptine and levodopa) does not prevent motor fluctuations in Parkinson's disease. Neurology 1993;43:21-7.

15 Factor SA, Weiner WJ. Early combination therapy with bromocriptine and levodopa in Parkinson's disease. Mov Disord 1993;8:257-62.
16 Montastruc JL, Rascol O, Senard JM, Rascol A. A randomised controlled study of bromocriptine versus levodopa in previously untreated parkinsonian patients: a 3 year follow-up. $\mathcal{F}$ Neurol Neurosurg Psychiatry 1989;52. 773-5.

17 Montrastruc JL, Rascol O, Rascol A. Comparaison de la bromocriptine et de la lévodopa dans le traitement de bromocriptine et de la levodopa dans le traitement de 3 ans d'une étude prospective randomisée. Rev Neurol 3 ans d'une étude prospec

18 Lees AJ. The relevance of Lewy body to the pathogenis of idiopathic Parkinson's disease. I Neurol Neurosurg Psychiatry 1988;51:745-52.

19 Golbe L, Davis P, Schenberg B, Duvoisin R. Prevalence and natural history of progressive supranuclear palsy. Neurology 1988;48:1031-4.

20 Quinn N. Multiple system atrophy: the nature of the beast. F Neurol Neurosurg Psychiatry 1989;(suppl):78-89.

$21 \mathrm{Gibb} W$, Lees A. A comparison of clinical pathological features of young- and old- onset Parkinson's disease. Neurology 1988;38:1402-6.

22 Hoehn M, Yahr M. Parkinsonism: onset, progression and mortality. Neurology 1967;17:427-42.

23 Fahn S, Elton $R$ and Unified Parkinson's Disease Rating Scale Development Committee. Unified Parkinson's disease rating scale. In: Fahn S, Marsden CD, Calne D, eds. Recent developments in Parkinson's disease. New York Macmillan 1987;2:153-63.

24 Obeso JA, Grandas F, Vaamonde J. Motor complications associated with chronic levodopa therapy in Parkinson's disease. Neurology 1989;39 (suppl 2):11-9.

25 Lees AJ. The on-off phenomenon. $\mathcal{f}$ Neurol Neurosurg Psychiatry 1989;(suppl):29-37.

26 Kaplan EL, Meier P. Non parametric estimation from incomplete observations. $\mathscr{f} \mathrm{Am}$ Stat Assoc 1958;53: 457-81.

27 Peto $R$, Pike MC, Armitage $P$, et al. Design and analysis of randomized clinical trials requiring prolonged observation of each patient. II. Analysis and examples. $\mathrm{Br} \mathcal{f}$ tion of each patient.

28 Tarone RE On summary estimations of relative risks. 7 Chron Dis 1981;34:463-8.

29 Mouradian MM, Heuser IJE, Baronti F, et al. Pathogenesis of dyskinesias in Parkinson's disease. Ann Neurol 1989;25:523-6.

30 Nutt JG. Levodopa-induced dyskinesia: review, observations and speculations. Neurology 1990;40:340-5.

31 Fabbrini G, Mouradian MM, Juncos $\mathrm{L}$, et al. Motor fluctuations in Parkinson's disease: central pathological mechanisms, part I. Ann Neurol 1988;24:366-71.

32 Nakanishi $T$, Kanazawa I, Iwata $M$, et al. Nation-wide collaborative study on the long-term effects of bromocriptine in the treatment of parkinsonian patients: analysis on the maintenance and the change of the original mode of the maintenance and the change of the original

33 Lees AJ, Stern GM. Sustained bromocriptine therapy in previously untreated patients with Parkinson's disease. previously untreated patients with Parkinson's

34 Bedard PJ, Gomez Mancilla B, Blanchette P, et al. Levodopa-induced dyskinesia: facts and fancy. What does the MPTP monkey model tell us? Can $\mathrm{f}$ Neurol Sci 1992;19:134-7.

35 Clemens JA, Fuller RW, Felten SY, Felten DL Neuroprotection. In: Agid Y, ed. Current trends in the treatment of Parkinson's disease. John Libbey and $\mathrm{Co}$ 1992:19-27.

36 Przuntek H, Welzel D, Blümmer E, et al. Bromocriptine lessens the incidence of mortality in levodopa-treated parkinsonian patients: prodo-study discontinued. Eur $f$ Clin Pharmacol 1992;43:357-9. 